\title{
Richtmyer-Meshkov turbulent mixing arising from an inclined material interface with realistic surface perturbations and reshocked flow
}

\author{
M. Hahn, ${ }^{1}$ D. Drikakis, ${ }^{1, a)}$ D. L. Youngs, ${ }^{2}$ and R. J. R. Williams ${ }^{2}$ \\ ${ }^{1}$ Department of Fluid Mechanics and Computational Science, Cranfield University, Cranfield MK43 OAL, \\ United Kingdom \\ ${ }^{2}$ AWE, Aldermaston, Reading, Berkshire RG7 4PR, United Kingdom
}

(Received 21 July 2010; accepted 25 February 2011; published online 26 April 2011)

\begin{abstract}
This paper presents a numerical study of turbulent mixing due to the interaction of a shock wave with an inclined material interface. The interface between the two gases is modeled by geometrical random multimode perturbations represented by different surface perturbation power spectra with the same standard deviation. Simulations of the Richtmyer-Meshkov instability and associated turbulent mixing have been performed using high-resolution implicit large eddy simulations. Qualitative comparisons with experimental flow visualizations are presented. The key integral properties have been examined for different interface perturbations. It is shown that turbulent mixing is reduced when the initial perturbations are concentrated at short wavelengths. The form of the initial perturbation has strong effects on the development of small-scale flow structures, but this effect is diminished at late times. [doi:10.1063/1.3576187]
\end{abstract}

\section{INTRODUCTION}

The Richtmyer-Meshkov instability (RMI) is derived from the works of Richtmyer ${ }^{1}$ and Meshkov ${ }^{2}$ on impulsively accelerated interfaces between two fluid layers with different densities caused by an incident shock wave. Initially, the instabilities develop rapidly and reach a nonlinear state where the mixing layer between heavy and light components exhibits characteristic mushroom-shaped bubbles and spikes. The flow development is associated with transition and turbulent mixing. An understanding of this mechanism for the amplification of the initial interface perturbations and the associated mixing process is important in relation to inertial confinement fusion (ICF), where RMI mixing is a critical factor in predicting the performance of ICF capsules. ${ }^{3-5}$ In ICF, a spherical capsule containing thermonuclear material is compressed using a powerful laser. ${ }^{4}$ Once a critical level of compression has been reached, the fuel ignites and a burst of energy is released. In ICF, RM instability occurs at the interface between the light and the heavy materials, triggering turbulent mixing. In this case, turbulent mixing has the dual effect of diluting and cooling the fuel, which reduces the efficiency of the reaction; hence, it is important that this mechanism is well understood. Other applications of RMI turbulent mixing include the wakes of jet engines and supersonic combustion, ${ }^{6}$ and the prediction of the evolution of supernovas. $^{7,8}$

Previous experimental investigations ${ }^{9-11}$ considered the flow physics of single-mode RMI. Two-dimensional computational studies, where the interface between the two gases was initialized by simple sinusoidal perturbations, were also performed. ${ }^{12,13}$ The numerical studies examined the growth

\footnotetext{
${ }^{a)}$ Author to whom correspondence should be addressed. Electronic mail: d.drikakis@cranfield.ac.uk.
}

rate of the mixing layer, mixing behavior, production of turbulent kinetic energy, and enstrophy.

In practical applications, however, the RichtmyerMeshkov instability encompasses multiple modes generated by random perturbations on the interface. These modes grow at different rates and lead to turbulent mixing. Additional complexities are encountered when shock wave reflections from the end wall of a shock tube or due to a "reshock" in the case of a spherical implosions, as observed in ICF capsules, occur. Limited experimental work has been carried out with respect to reshocked multimode RMI. The first experiment of this type was described by Andronov et al. ${ }^{14}$ The experiment of Vetter and Sturtevant ${ }^{15}$ has been simulated by Hill et al. ${ }^{16}$ and Schilling and Latini. ${ }^{17}$ In general, the numerical investigations agree well with the experimental results for the growth rate of the reshocked layer. However, the results for the initial growth of the mixing layer triggered by the passage of the incident shock wave are less satisfactory due to the dependence on the initial conditions, ${ }^{17,18}$ representing the surface perturbations between material interfaces. Furthermore, the evolution of Richtmyer-Meshkov instabilities and the growth of multiple modes introduced by random small-scale perturbations at the interface also depend on whether the impinging shock is locally inclined to the material surface because of geometrical constraints.

Due to the complexity of RMI for inclined shock waves, no analytical theory describing this problem has emerged yet. A previous experimental study considered a planar shock interacting with an inclined material interface. ${ }^{19}$ However, it is quite expensive to numerically reproduce the results because of the boundary layer effects associated with the presence of the solid walls in the experiment. In the series of shock tube experiments conducted at AWE, the influence of the walls on the diagnostics was reduced by using a lasersheet technique. The use of a fine wire grid to support the membrane initially used to separate the gases allowed for the 
modification of the initial interface geometry, resulting in two experiments with chevron ${ }^{20}$ and inverse chevron ${ }^{21}$ profiled configurations. The test case considered here is based on the inverse chevron experiment of Holder and Barton. ${ }^{21}$ Previous simulations of the same case were reported in Refs. 22 and 23. A similar experiment, using a double-bump perturbation, was described by Holder et al. ${ }^{24}$ In all of these experiments, the objective was to create a well-defined twodimensional flow on which fine scale turbulent mixing was superimposed, i.e., a flow that is two-dimensional on average and suitable for validation of two-dimensional Reynoldsaveraged Navier-Stokes models.

The aim of this paper is to investigate the growth of RMI and turbulent mixing under realistic conditions for the surface perturbations, including the effects of shock wave reflections. Simulations of the above problem require accurate capturing of the smaller modes in order to resolve the growth of the turbulent mixing region correctly. The computational study is based on the implicit large eddy simulation approach. ${ }^{25,26}$ The computational fluid dynamics code CNS3D (Refs. 26-28) has been employed, which has been previously applied to a wide range of flows featuring shock waves and turbulence. ${ }^{26,29-36}$ The remainder of this paper is organized as follows. Sections II and III present the governing equations and numerical methods employed in this study. The initial and boundary conditions for the inverse chevron test case are detailed in Sec. IV. Section V presents the results for the inverse chevron geometry for different interfacial perturbations. The conclusions are summarized in Sec. VI.

\section{GOVERNING EQUATIONS}

Richtmyer-Meshkov interactions typically occur at very high Reynolds number. In this limit, it is assumed that Re $\rightarrow \infty$ and the viscous terms in the Navier-Stokes equations are neglected. Thus, the Euler equations employed here in the context of multicomponent flows involve solving the momentum and energy equations in conjunction with the quasiconservative model of Allaire et al. ${ }^{37}$ The system of partial differential equations consists of two equations for the species densities

$$
\begin{aligned}
& \frac{\partial z_{1} \rho_{1}}{\partial t}+\nabla \cdot\left(z_{1} \rho_{1} \mathbf{u}\right)=0, \\
& \frac{\partial z_{2} \rho_{2}}{\partial t}+\nabla \cdot\left(z_{2} \rho_{2} \mathbf{u}\right)=0,
\end{aligned}
$$

momentum equation

$$
\frac{\partial \rho \mathbf{u}}{\partial t}+\nabla \cdot(\rho \mathbf{u} \otimes \mathbf{u}+P \mathbf{I})=0,
$$

the total energy equation

$$
\frac{\partial E}{\partial t}+\nabla \cdot[(E+P) \mathbf{u}]=0,
$$

and one equation for the volume fraction
TABLE I. Global variables of the fluid mixture [according to Allaire et al. (Ref. 37)].

\begin{tabular}{lc}
\hline \hline Variable & Description \\
\hline$z_{1}=z, z_{2}=1-z$ & Volume fractions \\
$\rho=z_{1} \rho_{1}+z_{2} \rho_{2}$ & Density \\
$y_{i}=z_{i} \rho_{i} / \rho$ & Mass fractions \\
$P=z_{1} P_{1}+z_{2} P_{2}$ & Pressure \\
$\rho e=z_{1} \rho_{1} e_{1}+z_{2} \rho_{2} e_{2}$ & Internal energy \\
$\rho h=z_{1} \rho_{1} h_{1}+z_{2} \rho_{2} h_{2}=\rho e+P$ & Specific enthalpy \\
$E=e+|\mathbf{u}|^{2} / 2$ & Specific total energy \\
$H=E+P / \rho$ & Specific total enthalpy \\
$\xi_{i}=1 /\left(\gamma_{i}-1\right)$ & Xi fractions \\
$\xi=z_{1} \xi_{1}+z_{2} \xi_{2}$ & Xi parameter \\
\hline \hline
\end{tabular}

$$
\frac{\partial z}{\partial t}+\mathbf{u} \cdot \nabla z=0,
$$

where $\rho, \mathbf{u}, E$, and $P$ are the densities, the velocity components, the total energy per unit volume, and the pressure, respectively. Furthermore, $\mathbf{I}$ is the identity tensor and $z=z_{1}$ is the volume fraction of air.

The above systems model the diffuse interface between compressible fluids where the global variables in the transition region can be defined by a mixture model or artificial equation of state. In order to avoid spurious acoustic waves near the spread interface and to maintain the necessary numerical stability of the method, an isobaric closure postulating equal phase and mixture pressures is employed. Furthermore, both fluids and the mixture are assumed to share the same velocity, and addition of enthalpy diffusion terms ${ }^{38}$ associated with numerical diffusion can be neglected.

In this paper, all fluids are modeled using an ideal gas equation of state given by

$$
P_{i}=\left(\gamma_{i}-1\right) \rho_{i} e_{i},
$$

with $\gamma$ and $e$ being the ratio of specific heats and internal energy, respectively, and $i$ stands for the individual fluids. For the mixture, the single $\gamma$-formulation is adopted where the coefficient depends on the volume fractions of the individual components. As reported in Ref. 39, to maintain an interface free from spurious pressure oscillations, it is necessary to calculate the averaged mixture coefficient $\gamma$ by introducing the auxiliary variable $\xi=1 /(\gamma-1)$. With this information, the thermodynamic parameters for the mixture are computed according to Table I.

Please note, albeit not being exploited in the current investigation, the model of Allaire et al. can be used in conjunction with any general equation of state. Additionally, the system of governing equations inherently obeys the phase mass and total energy conservation laws. For sharp material interfaces and/or dealing with ideal gases exclusively, the model could be reduced. However, in order to allow for future extension of the method, this paper features the more general framework. Details on the numerical approximation of the time derivatives and the flux calculation in the discretized form of the above equations are given in Sec. III. 


\section{NUMERICAL METHOD}

The numerical philosophy follows the implicit large eddy simulation (ILES) approach. This approach is based on the assumption that the specific features of the nonlinear numerical method locally and dynamically produce similar effects to explicit subgrid models used in conventional LES. All other assumptions are identical to the classical LES approach. Please note, when the current simulation method is applied to the compressible multicomponent system of equations, any dissipation or diffusion encountered during the solution process is purely numerical and does not necessarily represent the physical values encountered in experiments. Furthermore, it is assumed implicitly that turbulent mixing is driven by the resolved scales and Schmidt number effects are negligible.

The computational code CNS3D (Refs. 26-34) solves the governing equations using a finite volume Godunov-type method. ${ }^{40-42}$ The intercell numerical fluxes are computed based on the solution to the Riemann problem using the reconstructed variables at the left and right of the cell interface. The computational code includes a library of different Riemann solvers; ${ }^{31}$ however, in the present study, the Harten, Lax, van Leer, and (the missing) Contact (HLLC) approximate Riemann solver of $\mathrm{Toro}^{41}$ has been employed.

Higher order of accuracy in smooth flow regions is achieved using Monotone Upstream-centered Schemes for Conservation Laws (MUSCL) extrapolation, ${ }^{43}$ with the fifthorder limiter proposed by Kim and Kim. ${ }^{44}$ The standard MUSCL extrapolation has been augmented using a lowMach limiting scheme, ${ }^{45}$ which involves an additional stage in the reconstruction process for the velocity vector. It ensures uniform dissipation of kinetic energy in the limit of zero Mach number $(M)$, extending the validity of the Godunov method to at least $M \approx 10^{-4}$, via a progressive central differencing of the velocity components. The formulation of the underlying governing equations is not changed, and monotonicity of the density and scalar field is maintained.

It was shown in Ref. 45 that the leading order kinetic energy dissipation rate is proportional to $u^{3} / \Delta x$ for the modified scheme, similar in form to that proposed by Kolmogorov $^{46}$ for decaying turbulence. It is this dissipation rate that acts as a subgrid model in the ILES framework, giving significantly improved high wavenumber performance compared to standard Godunov methods. ${ }^{45}$ Although this analytical justification is only indicative of the capabilities of the numerical framework, the conclusion is strongly supported by successful application of the method to various cases. ${ }^{32,33,47-50}$

Previously, numerical investigations have been performed for Richtmyer-Meshkov mixing using ninth-order weighted essentially nonoscillatory (WENO) methods. ${ }^{32,33}$ However, when used in combination with the modified lowMach limiting method, the scheme leads to significant turn-up at the high wavenumber end of the energy spectrum. This indicates that the modified WENO method produces slightly less numerical dissipation as would be expected from a classical subgrid scale model. The modified MUSCL scheme, however, does not exhibit the strong turn-up and

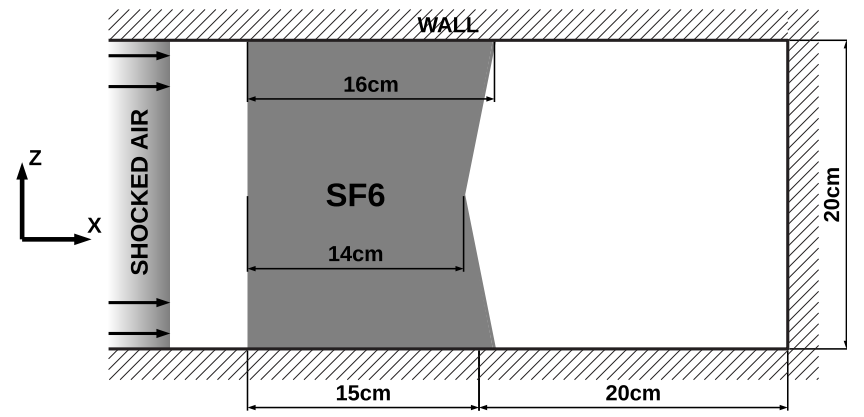

FIG. 1. Schematic of the inverse chevron configuration.

leads to the correct physical behavior. Therefore, in the present study the fifth-order limiter was selected. as a betterunderstood scheme for this type of flow. The time integration of Eqs. (1a)-(1e) has been obtained using a three-stage total variation diminishing Runge-Kutta method. ${ }^{42}$

\section{INITIAL AND BOUNDARY CONDITIONS}

Figure 1 illustrates the quasi-two-dimensional setup featuring a block of the dense gas sulfur hexafluoride $\left(\mathrm{SF}_{6}\right)$ encased in air. The case considered here is based on the inverse chevron experiment of Holder and Barton. ${ }^{21}$ As an incident shock wave of Mach 1.26 passes from left to right, the membranes separating the two fluids rupture and Richtmyer-Meshkov instabilities are formed. The membranes are supported on a very fine wire mesh with a grid spacing of $0.4 \mathrm{~cm}$. The initially small perturbations grow rapidly in size, leading to high Reynolds number turbulent mixing of the two gases. This effect is amplified further by the existence of wave reflections from the material interfaces and the end wall of the shock tube.

The boundary conditions in the simulations are reflective on the upper, lower, and end walls. In the streamwise direction, an extended one-dimensional domain has been employed to minimize spurious reflections from the upstream outflow boundary. The computational domain extends $10 \mathrm{~cm}$ in the lateral direction where periodic conditions are applied. The use of periodic boundary conditions, rather than reflective boundary conditions, in the lateral direction gives a flow which is statistically homogeneous in the z-direction and better suited to mathematical analysis. The results obtained should be suitable for comparison with the midplane lasersheet images. Initially, $\mathrm{SF}_{6}$ and air are in pressure equilibrium at 1 bar with densities of 6.34 and $1.184 \mathrm{~kg} \mathrm{~m}^{-3}$, respectively, giving an Atwood number of 0.68. Furthermore, temperature equilibrium has been assumed, yielding specific heat ratios of 1.076 for $\mathrm{SF}_{6}$ and 1.4 for air. The $\mathrm{SF}_{6}$ is seeded with olive oil droplets for the laser-sheet diagnostic. Its density has been increased slightly according to the proportions of the individual components in the mixture to allow for this. The shock wave is modeled as compressed air moving with a velocity of $136.87 \mathrm{~m} \mathrm{~s}^{-1}$ under a pressure of 1.706 bars and a density of $1.7264 \mathrm{~kg} \mathrm{~m}^{-3}$.

In the experiments, the wire mesh supporting the membranes is assumed to be responsible for the small-scale instabilities at the initial surface between the two gases. In order 

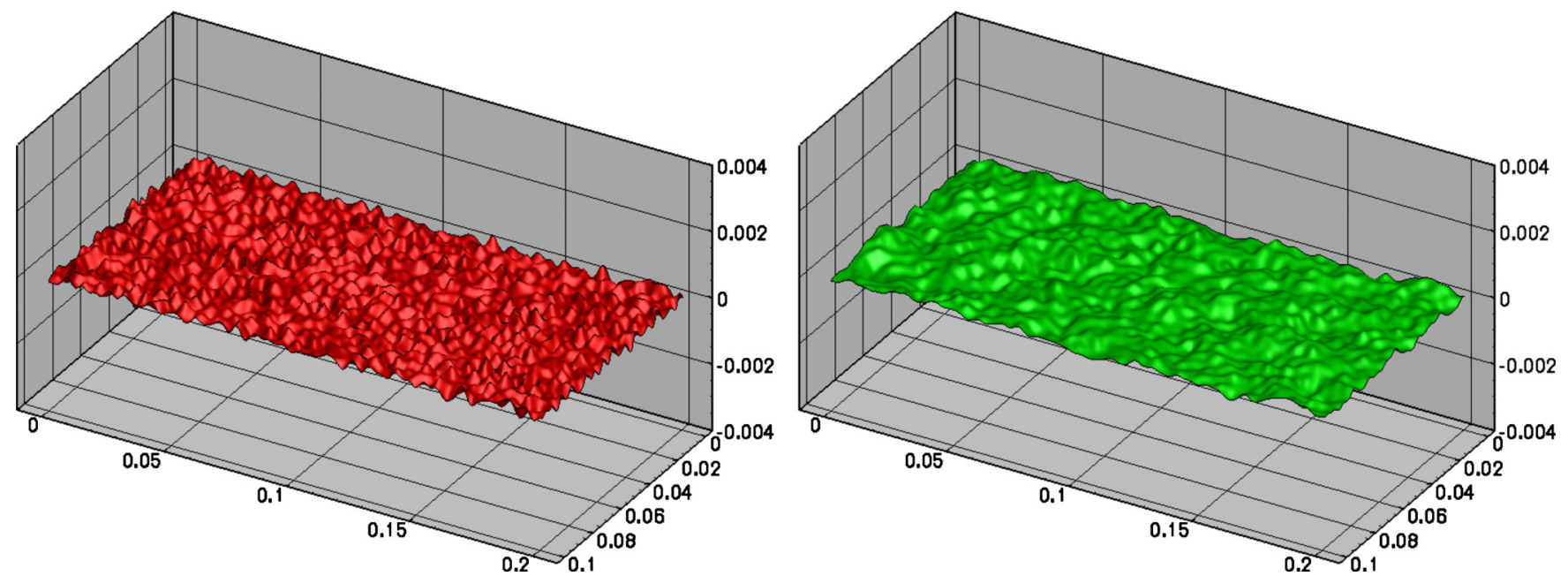

FIG. 2. (Color online) Initial interface perturbations (left: $k$-spectrum; right: $k^{-2}$-spectrum).

to produce a realistic material interface, it is important to model the initial perturbations accurately as they have a significant influence on the results of the simulations.

A realistic method of representing a two-dimensional interface between two materials is the summation of a number of individual wave modes in accordance with an assumed surface perturbation power spectrum as proposed by Youngs. ${ }^{51}$ The left and right interface perturbations are given by

$$
\begin{aligned}
\zeta_{R}(y, z)= & S \sum_{m, n=0}^{N}\left[a_{m, n} \cos \left(k_{y 0} n y\right)+b_{m, n} \sin \left(k_{y 0} n y\right)\right] \\
& \times \cos \left(k_{z 0} m z\right),
\end{aligned}
$$

where $k_{y 0}=2 \pi / W_{y}, \quad k_{z 0}=\pi / W_{z}, \quad W_{y}=10 \mathrm{~cm}, \quad$ and $W_{z}$ $=20 \mathrm{~cm}$. The amplitudes $a_{m, n}$ and $b_{m, n}$ are chosen from a Gaussian distribution provided that the wavelength $\lambda$ $=2 \pi / \sqrt{n^{2} k_{y 0}^{2}+m^{2} k_{z 0}^{2}}$ is in the range between $\lambda_{\min }=0.5 \mathrm{~cm}$ and $\lambda_{\max }=5.0 \mathrm{~cm}$, otherwise they are zero. Furthermore, a scaling factor $S$ is applied to achieve a standard deviation of $\sigma=0.01 \mathrm{~cm}$ for $\zeta_{R}(y, z)$. These parameters are chosen according to the spacing of the wire-frame membrane and the physical dimensions of the shock tube.

In this investigation, interface perturbations satisfying a power spectrum of the form $P(k) \propto k^{-2}$, where $\sigma^{2}=\int P(k) d k$ $=\left\langle\zeta^{2}(k)\right\rangle$, are used to represent a typical surface finish found for inertial confinement fusion capsules. ${ }^{52}$ For the $k^{-2}$-spectrum, the perturbations are concentrated at long wavelengths and the results are compared against simulations using a power spectrum proportional to $k$, where the majority of the perturbations are accumulated at short wavelengths (Fig. 2), as used in Ref. 24 to model membrane rupture. Additionally, two different grid resolutions of $\Delta$ $=1 / 16 \mathrm{~cm}$ and $\Delta=1 / 32 \mathrm{~cm}$ with $640 \times 160 \times 320$ and $1280 \times 320 \times 640$ grid nodes, in $(x, z, y) \quad(y$ is the periodic direction), respectively, are considered in order to assess grid convergence.

\section{RESULTS}

\section{A. Flow development}

The flow development resulting from two different perturbation spectra can be discussed qualitatively on the basis of the volume fraction contours presented in Figs. 3 and 4. Here, snapshots taken at identical times for a grid resolution of $\Delta=1 / 16 \mathrm{~cm}$ are shown for the initial $k$ (left column) and $k^{-2}$ spectra (right column).

In general, the evolution of the flow is similar in both cases. The initial conditions as the shock wave impacts on the left-hand interface $(t=0 \mathrm{~ms})$ have been visualized in the first row of Fig. 3. After shock passage, the flow at the first interface starts moving toward the end wall and the perturbations grow (row 2). After $1.3 \mathrm{~ms}$, the shock has already passed through the second interface, has reflected from the end plate, and is about to interact with the right-hand interface for the second time. Note that the characteristic shape inversion of the chevron geometry caused by the change from heavy to light fluid is clearly visible in both cases. The $\mathrm{SF}_{6}$ slab is, subsequently, decelerated as the reflected shock passes through, between $t=1.3 \mathrm{~ms}$ (last row in Fig. 3) and $t=2.2 \mathrm{~ms}$ (first row in Fig. 4), and the perturbation growth is amplified. After this stage, the interaction with several reflected waves keeps exciting the development of the layers of the material interface and turbulent mixing occurs at later times. Furthermore, a large spike has been ejected along the centerline and starts rolling up into a vortex. This most prominent flow feature continues to travel toward the end wall until it finally impinges on and subsequently spreads along it.

Several differences between the two simulations can be observed. As intended, the $k^{-2}$-spectrum lacks the small-scale perturbations observed for the $k$-spectrum (first row in Fig. $3)$. Consequently, the initial growth of the mixing layer between the two fluids is relatively slow. After the reshock has passed the $\mathrm{SF}_{6}$-region (first row in Fig. 4), more large-scale activity is still present for the $k^{-2}$-spectrum. This has a sig- 

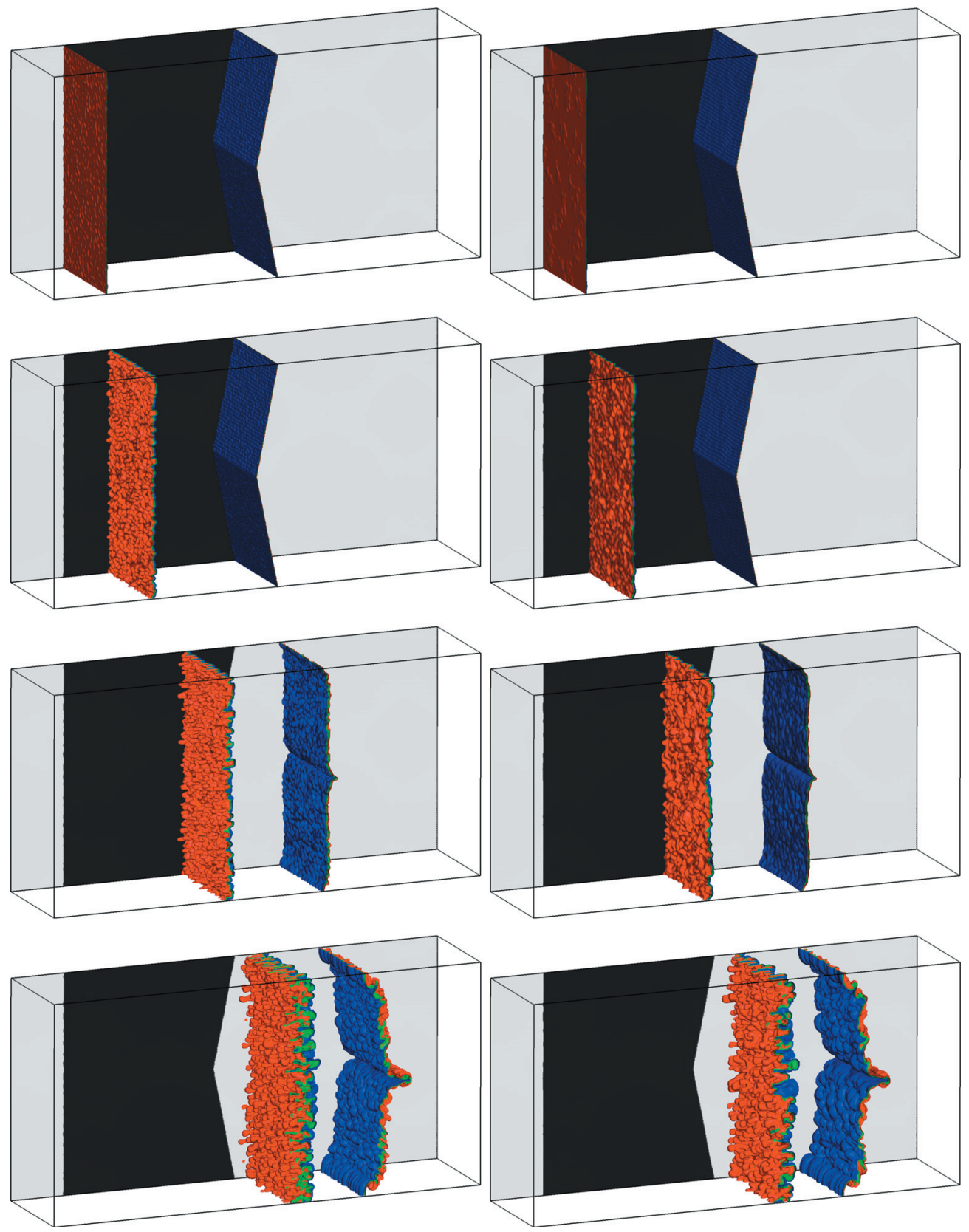

FIG. 3. (Color) Development of the flow visualized by contours of volume fraction (left: $k$-spectrum; right: $k^{-2}$-spectrum; top to bottom: snapshots at times $0.0,0.5,1.3$, and $1.9 \mathrm{~ms})$.

nificant effect on the subsequent turbulent mixing as the large-scale structures do not break down as fast as for the $k$-spectrum. Thus, a larger bubble roll-up is observed near the top and bottom walls and more fluid entrainment is observed (row 3 in Fig. 4). This leads to a strong rotation of the mixing layer situated between the bubbles and the spike compared to the case of $k$, especially at the bottom half of the shock tube (last row in Fig. 4).

\section{B. Comparisons with the experiment}

Turbulent mixing in the inverse chevron experiments ${ }^{21}$ has been visualized using pulsed laser-sheet illumination of the Mie scattered light from seeding particles in the $\mathrm{SF}_{6}$ region. In the simulations, the $\mathrm{SF}_{6}$ mass per unit volume can be interpreted as the numerical equivalent of the photographic images. Note that as explained in Ref. 24, the laser-sheet technique is subject to multiple scattering of the reflected light, thus the experimental pictures appear slightly diffuse and only the large-scale features can be observed. As the flow is essentially two dimensional at the largest scales, the line average (y-direction) of the $\mathrm{SF}_{6}$ density is shown here.

The development of the inverse chevron flow at different time instants is shown in Fig. 5 for the experiment and simulations, for two different initial conditions, using the $\mathrm{SF}_{6}$ density plots. After $1.9 \mathrm{~ms}$, from the time when the initial shock wave impacts on the left interface between air/ $\mathrm{SF}_{6}$, inversion of the right interface and the occurrence of the prominent central spike discharging along the centerline are 

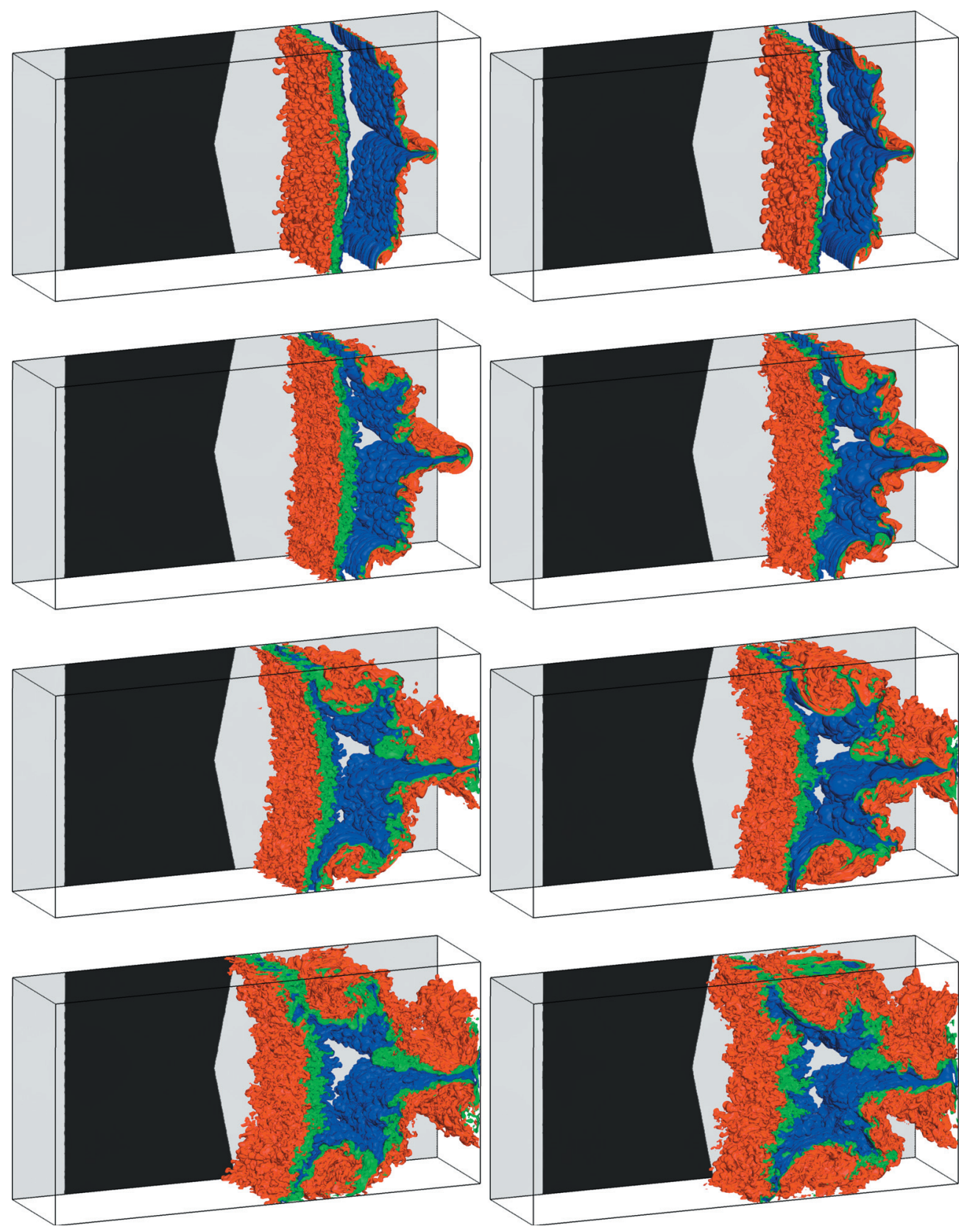

FIG. 4. (Color) Development of the flow visualized by contours of volume fraction (left: $k$-spectrum; right: $k^{-2}$-spectrum; top to bottom: snapshots at times $2.2,2.7,3.3$, and $4.0 \mathrm{~ms}$ ).

observed. The bubbles at the top and bottom walls of the shock tube do not grow until $2.7 \mathrm{~ms}$ (second column in Fig. 5). In the late stages and similar to the experiment, the central spike in the simulations impacts on and spreads along the end wall and the bubbles roll-up into two large-scale vortical structures.

Additionally, measurements of the position of the $\mathrm{SF}_{6}$ block at early times have been taken from the photographic images obtained in the experiments. Figure 6 compares the experimental and numerical data after $1.9 \mathrm{~ms}$ by superimposing the measured locations (light gray lines) onto the $\mathrm{SF}_{6}$ density contours predicted in the simulations. For this purpose, three contour levels of $0.5,1.45$, and 2.25 times the initial $\mathrm{SF}_{6}$ density have been chosen, where the first two identify the position of the $\mathrm{SF}_{6}$ block and the last one relates to the shock location. In general, good qualitative agreement between the experiment and the simulations is observed.

A further quantitative comparison between the simulations and the experiment is presented in Fig. 7 for the growth of the most prominent flow features. The location of the wall-bubble left edge and the tip of the spike have been extracted from the simulations by tracking the values of volume fraction 0.5 and 0.1 , respectively. The positions shown in Fig. 7 represent the associated distances from the end wall of the shock tube, which have been taken from the experimental photographs. Both the experiment and the simulations show that the spike approaches the end wall and impinges on it, after approximately $3 \mathrm{~ms}$, and further starts spreading. Good agreement between the experiment and the simulations has also been obtained for the position of the 

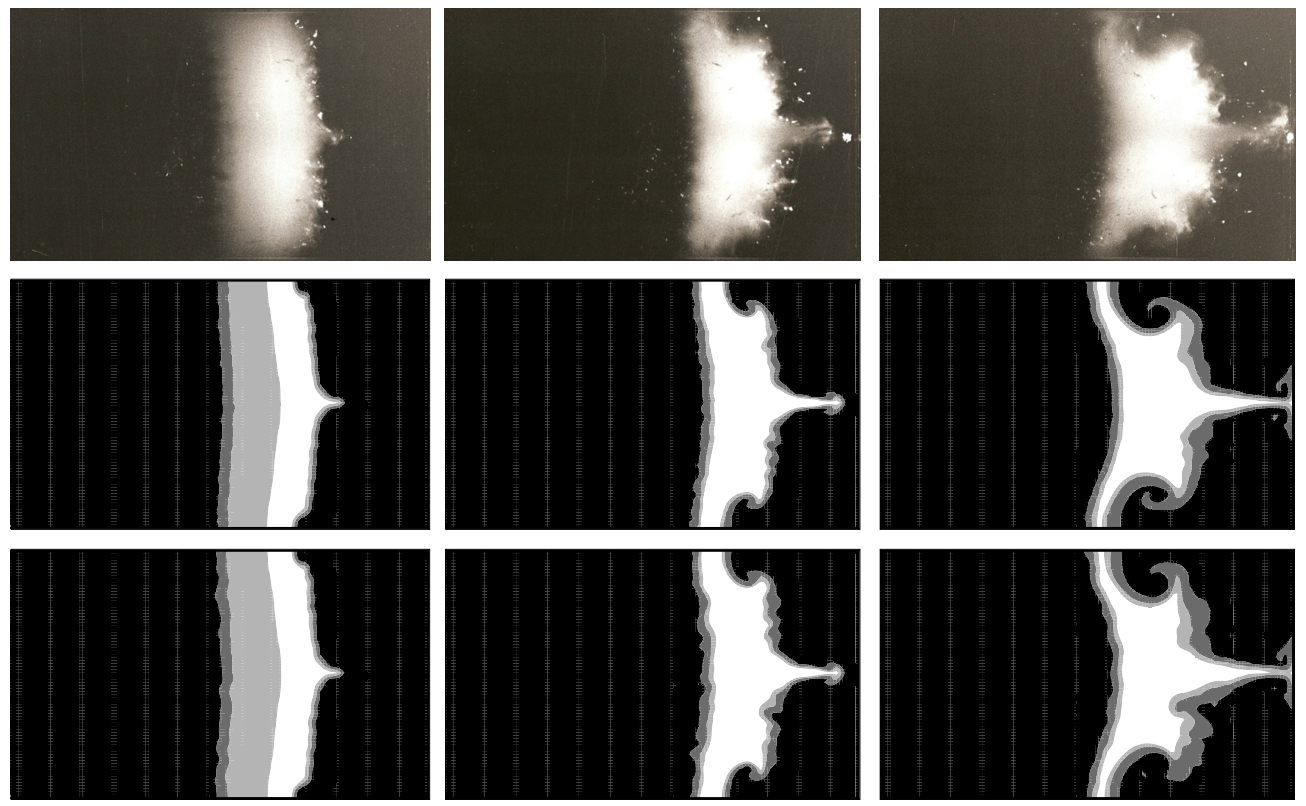

FIG. 5. (Color online) Comparison of the flow development between experiment and simulation (left to right: snapshots at times 1.9, 2.7, and 3.3 ms; top to bottom: experiment, $k$-spectrum, $k^{-2}$-spectrum).

bubble. The locations extracted from experiment are subject to uncertainties associated with the interpretation of the photographs, especially for the bubble location at late times. Additionally, the bubble position toward the end of the simulation is not expected to be a good match with the experimental values as viscous wall effects are neglected in the computations. Please note both, essentially two-dimensional, large-scale features are not very sensitive to the characteristics of the small-scale perturbations. In fact, reducing the complexity of the simulation by restricting it to two dimensions and removing any additional perturbation leads to almost identical results. However, if one is interested in the turbulence characteristics generated by the small-scale perturbations, it is absolutely essential to consider the fully three-dimensional flow.

\section{Integral parameters}

The influence of the form of the initial perturbations on key integral parameters is shown in this section. Note that the left domain boundary is simply chosen sufficiently far from the region of interest in order not to affect the results and the integral parameters used here are defined so that they are also unaffected by the left-hand boundary position.
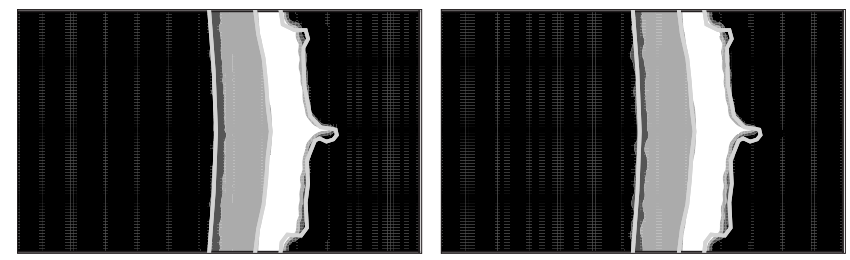

FIG. 6. Comparison of the $\mathrm{SF}_{6}$ location between experiment (light gray lines) and contour plots obtained in the simulations after $1.9 \mathrm{~ms}$ (left: $k$-spectrum; right: $k^{-2}$-spectrum).
First, the potential for hypothetical chemical reactions is investigated. Consider a hypothetical rate-determined chemical reaction between species A, initially in fluid 1, and species $\mathrm{B}$, initially in fluid 2 , with uniform molar concentrations per unit mass $c_{1}$ and $c_{2}$, respectively. As the fluids mix, it is assumed that both (i) diffusion of $\mathrm{A}$ and $\mathrm{B}$ relative to the initial fluids and (ii) depletion of A and B are small. The total reaction rate is then given by

$$
\mathrm{R}=\int k(T)[\mathrm{A}][\mathrm{B}] d V=\int k(T) c_{1} c_{2} \rho^{2} m_{1} m_{2} d V
$$

where $m_{1}$ and $m_{2}$ are the mass fractions of $\mathrm{A}$ and $\mathrm{B}$, respectively, and $\rho$ is the total density. The reaction rate constant $k$ will, in general, vary with temperature $T$. However, if the variation is neglected, the total reaction rate is proportional to the quantity

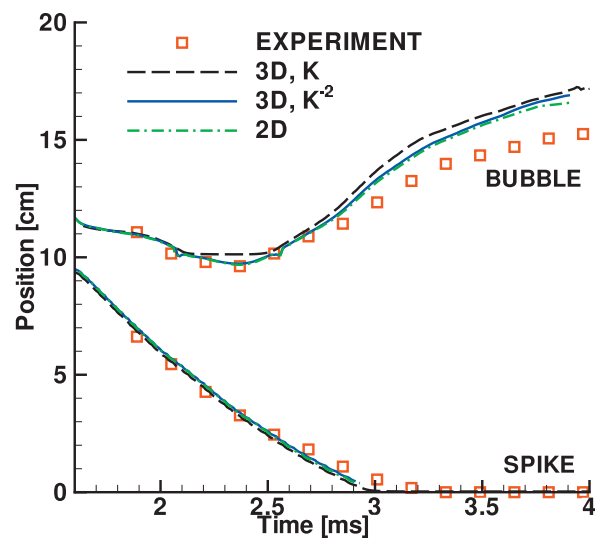

FIG. 7. (Color) Comparison of the bubble and spike positions between experiment and simulations for a grid resolution of $\Delta=1 / 16 \mathrm{~cm}$. 


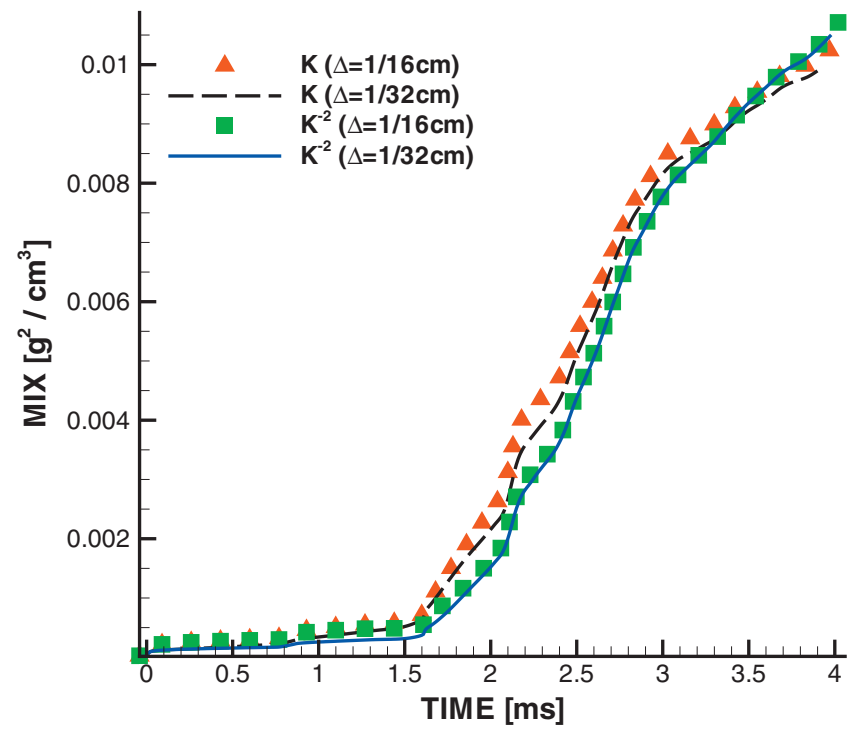

FIG. 8. (Color) Evolution of the total mixing parameter vs time for different perturbation spectra and grid resolutions.

$$
\mathrm{MIX}=\int \rho^{2} m_{1} m_{2} d V .
$$

This integral quantity is not susceptible to statistical fluctuations and gives a simple way of measuring variations in the total amount of mixing in a complex flow.

In Fig. 8, the effect of mesh size and initial perturbations on the integral parameter MIX is shown. In all simulations, the passage of the incident and reflected shocks is marked by a sudden increase in the level of mixing up to a time of approximately $2.6 \mathrm{~ms}$. Furthermore, the results for the $k^{-2}$-spectrum appear to be better converged than the $k$-spectrum, at least with reference to the total mixing. This can be explained by the lack of small-scale perturbations in the case of $k^{-2}$. However, this would require running higher grid resolutions before grid convergence is confirmed.

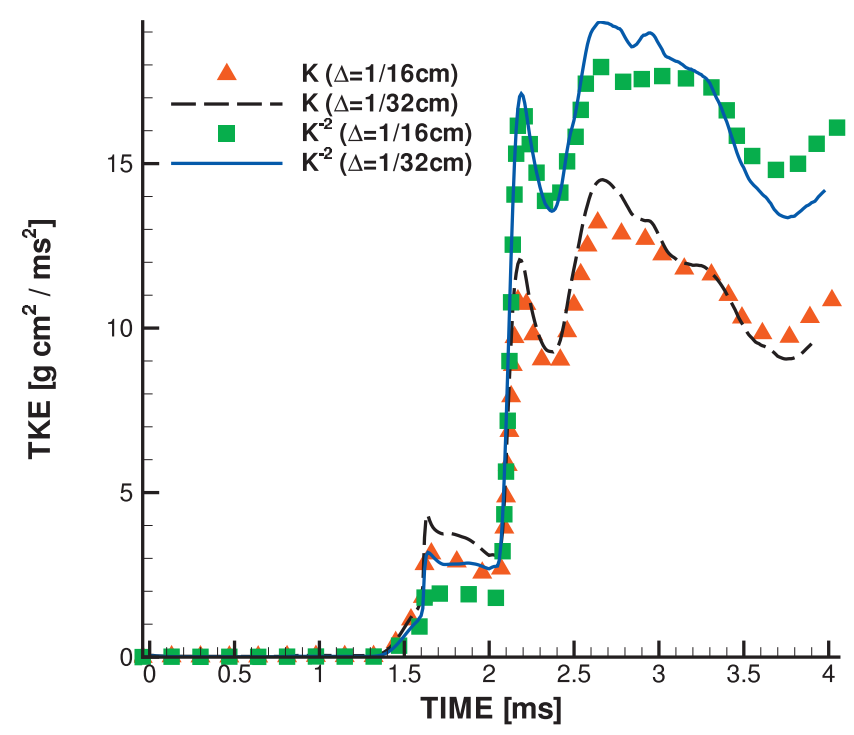

At the initial RMI stages, the $k$-spectrum results in a slightly more mixing compared to the $k^{-2}$-spectrum (Fig. 8). After approximately $1.5 \mathrm{~ms}$, when the reshock interacts with the $\mathrm{SF}_{6}$ slab, the differences in the mixing behavior obtained with the two spectra are evident. As the mixing layers develop faster in the case of the $k$-spectrum, a higher amount of total mixing is observed compared to $k^{-2}$. Only at very late times the flow entrainment into the large vortices near the walls and the higher energy modes generated by the $k^{-2}$-spectrum lead to more turbulent mixing and, consequently, the total amount of mixing for the $k^{-2}$-spectrum is higher than the $k$-spectrum.

The second key parameter examined is the total turbulent kinetic energy (TKE). As noted earlier, the experiment features an inclined material interface which is two dimensional. Hence, the essentially two-dimensional "background flow" is removed when considering the turbulence statistics by first calculating a two-dimensional turbulent kinetic energy field. This is achieved through averaging in the $y$-direction and then the required parameter is obtained by integration over the whole volume as follows:

$$
\mathrm{TKE}=\int \rho k d V,
$$

where

$$
k=\left\langle\frac{1}{2} \rho\left(\left(u_{x}-\tilde{u}_{x}\right)^{2}+\left(u_{z}-\tilde{u}_{z}\right)^{2}+u_{y}^{2}\right)\right\rangle /\langle\rho\rangle
$$

and

$$
\tilde{u}_{x}=\left\langle\rho u_{x}\right\rangle /\langle\rho\rangle, \quad \tilde{u}_{z}=\left\langle\rho u_{z}\right\rangle /\langle\rho\rangle .
$$

Here, \langle\rangle denotes the two-dimensional average in a plane normal to the lateral y-direction.

The evolution of TKE is shown in Fig. 9, where the passage of incident and reflected shocks is indicated by sudden jumps in the TKE values. Note that the increase of TKE caused by the interaction with the incident shock at times less than $1 \mathrm{~ms}$ is not visible on the current plotting scale (left

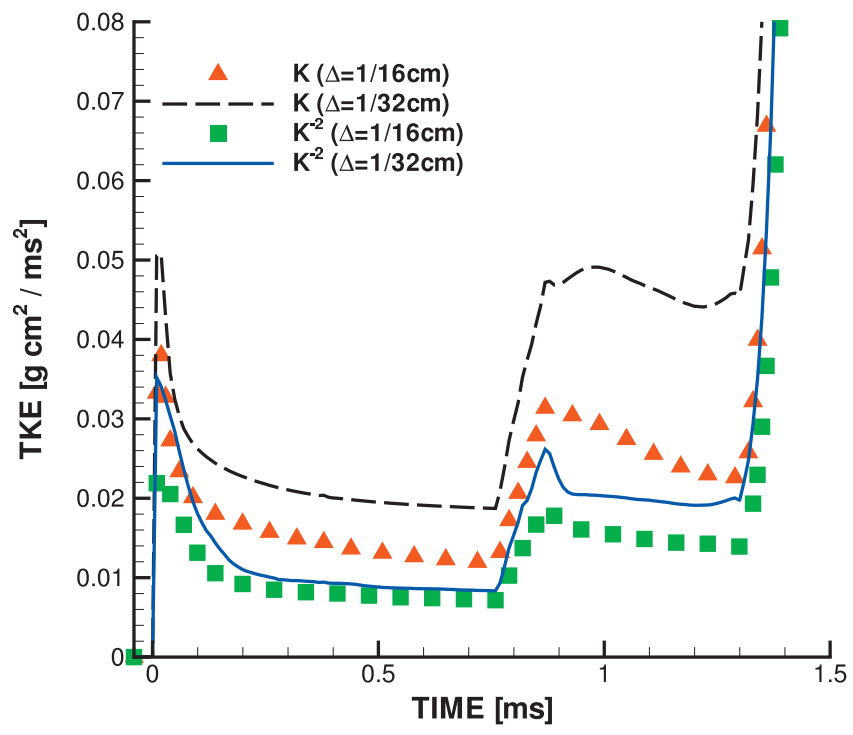

FIG. 9. (Color) Evolution of total turbulent kinetic energy for different perturbation spectra and grid resolutions. 

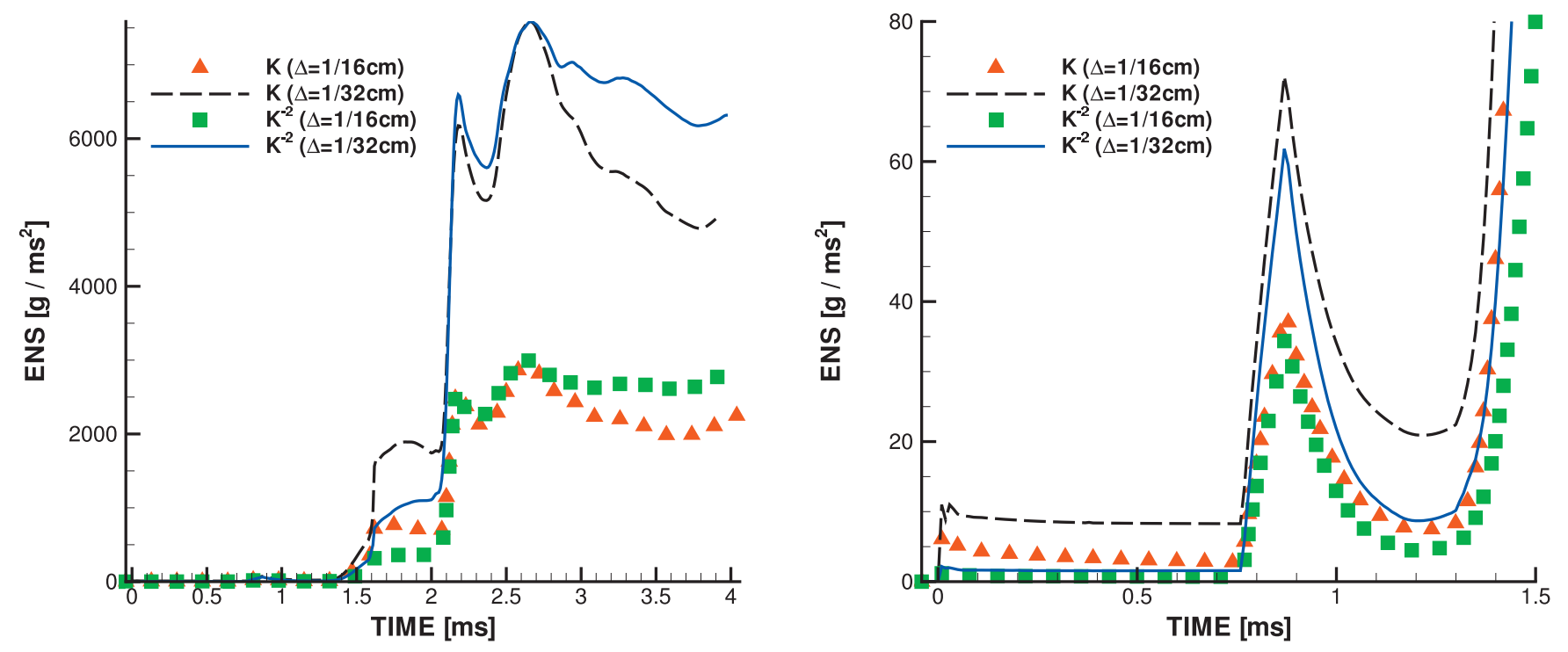

FIG. 10. (Color) Evolution of total enstrophy vs time for different perturbation spectra and grid resolutions.

plot). Therefore, the first (visible) production of TKE is shown as the reflected shock wave impacts on the right-hand interface of the $\mathrm{SF}_{6}$ region at approximately $1.5 \mathrm{~ms}$. Since this interface features the inverse chevron geometry, the increase at $1.5 \mathrm{~ms}$ is not a sharp jump but is rather gradual. With respect to the TKE jump at $1.5 \mathrm{~ms}$, the $k$-spectrum encompasses more TKE than the $k^{-2}$-spectrum (compared on the same grid). This may be due to the faster mixing layer development of the $k$-spectrum. However, this effect can be compensated for by increasing the grid resolution, as demonstrated by the results obtained on the finer mesh.

Looking at early time when the incident shock passes through the two interfaces (Fig. 9, right plot), more turbulent kinetic energy is produced for the $k$-spectrum because it contains more small-scale energetic perturbations compared to $k^{-2}$. Because the less-energetic small-scale perturbations are resolved better when refining the grid $\left(k^{-2}\right.$-spectrum), a higher increase in the TKE is observed at early times (Fig. 9, right plot). The TKE at the planar interface decays subsequently to a fairly similar level for both grids. However, the early time development of turbulent kinetic energy also reveals that the limits of the numerical method are being pushed on the coarse grid in terms of resolution power, especially for the $k$-spectrum.

A somewhat different picture is shown for the interaction with the inclined interface at approximately $0.9 \mathrm{~ms}$, where the TKE is not grid-converged. The TKE just before reshock (at $1.3 \mathrm{~ms}$ ) is the same for the $k^{-2}$-spectrum (fine grid) and $k$-spectrum (coarse grid). This behavior can be explained by the additional energy associated with better grid resolution of the Kelvin-Helmholtz type roll-ups at the inclined interface for the $k^{-2}$-spectrum. This is also confirmed by the fine grid simulation using the $k$-spectrum. As a result, the postreshock TKE on the fine grid in case of $k^{-2}$ is equivalent to the TKE level obtained on the coarse grid for the $k$-spectrum.

The most prominent increase in the TKE (Fig. 9, left plot) coincides with two events occurring after $2.1 \mathrm{~ms}$ : the primary reflected shock and a secondary shock reflection passing through the left- and the right-hand interfaces, respectively. Therefore, a strong amplification of TKE is visible at this point in time. The difference in the TKE jump between the spectra cases is due to the excitation of different modes present in the mixing zone. As the $k^{-2}$-spectrum leads to more large-scale features, more turbulent kinetic energy is contained in the flow after the passage of the shocks. Note that the initial shock amplifies the initial perturbations and sets the "seed" for the main mixing process, which is due to the reflected shocks. Although the high wavenumber component of this seed is not fully grid-converged, it is reasonable to assume that the final values of TKE depend only weakly on it. This can be explained by the nonlinear nature of the multiply shock process and by the fact that the low wavenumber component of the seed, which has a greater significance at late times, is well resolved. Similar conclusions can be found in Ref. 18. Therefore, after $2.1 \mathrm{~ms}$, the TKE results are expected to be better converged and are assumed to be accurate despite the slightly under-resolved early time development. Finally, when the secondary shock reflection passes through the right-hand interface at approximately $2.5 \mathrm{~ms}$, a further increase of TKE occurs.

An additional integral parameter considered here is the mass-weighted total enstrophy,

$$
\Omega=\int \rho(\nabla \times \mathbf{u})^{2} d V
$$

This quantity has no converged values, but is expected to increase with grid resolution. High levels of enstrophy indicate many small energetic vortices, thus it can be used as a measure of the small scales present in the flow. If the turbulent kinetic energy has a $k^{-5 / 3}$ Kolmogorov spectrum, then the enstrophy should have a $k^{1 / 3}$ spectrum. Therefore, integration up to a grid-related cutoff therefore suggests that $\Omega$ $\propto \Delta^{-4 / 3} \cdot 22$

Figure 10 shows the evolution of total enstrophy during the entire course of the simulations (left) and a close-up of 
the development prior to reshock (right). As expected, when the incident shock wave impacts on the planar interface at 0 $\mathrm{ms}$, more enstrophy is produced from the $k$-spectrum because it contains more small-scale turbulent structures than the $k^{-2}$ spectrum. Maintaining the form of the spectrum and increasing the grid size lead to higher values because the small-scale perturbations are better resolved on a finer grid. For the shock interacting with the inclined interface at approximately $0.9 \mathrm{~ms}$, the grid resolution has an even more pronounced effect on the enstrophy production. It outweighs the influence of the initial spectrum as seen by the high peaks for both spectra with a grid spacing of $\Delta=1 / 32 \mathrm{~cm}$ on the righthand side in Fig. 10. However, a sharp decrease in enstrophy can also be observed as the shock passes the inclined interface. Lower levels of enstrophy are apparent after reshock at $1.6 \mathrm{~ms}$ in the case of $k^{-2}$ when compared to $k$ on the same grid. The use of a finer grid compensates for this effect and leads to similar values with the $k$-spectrum on the coarse grid. However, because the small scales introduced by the perturbations are already in the limit of the grid resolution $\Delta=1 / 16 \mathrm{~cm}$, the enstrophy levels off in the coarse grid simulations. In contrast, a finer mesh allows for the vortex dynamics to act on the small scales and, consequently, the enstrophy keeps increasing. After $2.1 \mathrm{~ms}$, when both the primary and the secondary reflected shocks cause the large increase in enstrophy, a grid-dependent enstrophy level emerges irrespective of the initial state prior to the interaction. During the late-time development, the enstrophy generated from the $k$-spectrum appears to slightly drop because the flow lacks the large energetic scales that is fed upon. Halving the mesh size increases the peak value of enstrophy by a factor of 2.5 , which is very close to a value of 2.52 predicted by the $-4 / 3$ power scaling discussed above. It is interesting to note that the difference in enstrophy by a large factor corresponds to only small differences in the total amount of mixing, which confirms that mixing is driven mainly by the resolved larger scales.

\section{CONCLUSIONS}

The flow physics associated with the passage of a shock wave, including reshocked flow, through an inclined material interface with perturbations with different spectra but the same variance, has been investigated. The test case considered here has been based on the experimental work of Holder and Barton. ${ }^{21}$

Short wavelength surface irregularities approximated by a power spectrum proportional to the wavenumber of the mode lead to more total mixing in the early stages, but cannot maintain the turbulent mixing rate at late times due to the lack of long-living large energetic scales. Additionally, the turbulent kinetic energy decays faster after shock interaction with the inclined interface when compared to long wavelength surface irregularities characterized by a spectrum of the form $P(k) \propto k^{-2}$.

Initially, the total amount of small-scale structures present in the flow, as quantified by the integral value of enstrophy, is sensitive to the perturbation spectrum. As the flow evolves, however, a level independent of the initial condition emerges and the late-time development is dominated by the total amount of TKE.

\section{ACKNOWLEDGMENTS}

The AWE's financial support to Dimitris Drikakis through the William Penney Fellowship is gratefully acknowledged. The manuscript contains material (c) British Crown Copyright 2011/MoD, reproduced with permission.

${ }^{1}$ R. D. Richtmyer, "Taylor instability in shock acceleration of compressible fluids," Commun. Pure Appl. Math. 13, 297 (1960).

${ }^{2}$ E. E. Meshkov, "Instability of the interface of two gases accelerated by a shock wave," Fluid Dyn. 43, 101 (1969).

${ }^{3}$ R. L. Holmes, G. Dimonte, B. Fryxell, M. L. Gittings, J. W. Grove, M. S. Schneider, D. H. Sharp, A. L. Velikovich, R. P. Weaver, and Q. Zhang, "Richtmyer-Meshkov instability growth: Experiment, simulation and theory," J. Fluid Mech. 389, 55 (1999).

${ }^{4}$ P. Amendt, J. D. Colvin, R. E. Tipton, D. E. Hinkel, M. J. Edwards, O. L. Landen, J. D. Ramshaw, L. J. Suter, W. S. Varnum, and R. G. Watt, "Indirect-drive noncryogenic double-shell ignition targets for the National Ignition Facility: Design and analysis," Phys. Plasmas 9, 2221 (2002).

${ }^{5}$ M. Brouillette, "The Richtmyer-Meshkov instability," Annu. Rev. Fluid Mech. 34, 445 (2002).

${ }^{6}$ J. Yang, T. Kubota, and E. Zukoski, "Applications of shock-induced mixing to supersonic combustion," AIAA J. 31, 854 (1993).

${ }^{7}$ W. D. Arnett, J. N. Bahcall, R. P. Kirshner, and S. E. Woosley, "Supernova 1987A," Annu. Rev. Astron. Astrophys. 27, 629 (1989).

${ }^{8}$ A. S. Almgren, J. B. Bell, C. A. Rendleman, and M. Zingale, "Low Mach number modeling of type Ia supernovae. I. Hydrodynamics," Astrophys. J. 637, 922 (2006).

${ }^{9}$ M. Brouillette and B. Sturtevant, "Experiments on the RichtmyerMeshkov instability: Single-scale perturbations on a continuous interface," J. Fluid Mech. 263, 271 (1994).

${ }^{10}$ B. D. Collins and J. W. Jacobs, "PLIF flow visualization and measurements of the Richtmyer-Meshkov instability of an air/SF ${ }_{6}$ interface," J. Fluid Mech. 464, 113 (2002).

${ }^{11}$ J. W. Jacobs and V. V. Krivets, "Experiments on the late-time development of single-mode Richtmyer-Meshkov instability," Phys. Fluids 17, 034105 (2005).

${ }^{12}$ M. Latini, O. Schilling, and W. S. Don, "Effects of WENO flux reconstruction order and spatial resolution on reshocked two-dimensional Richtmyer-Meshkov instability," J. Comput. Phys. 221, 805 (2007).

${ }^{13}$ O. Schilling, M. Latini, and W. S. Don, "Physics of reshock and mixing in single model Richtmyer-Meshkov instability," Phys. Rev. E 76, 026319 (2007).

${ }^{14}$ V. A. Andronov, S. M. Bakhrakh, E. E. Meshkov, V. N. Mokhov, V. V. Nikiforov, A. V. Pevnitskii, and A. I. Tolshmyakov, "Turbulent mixing at contact surface accelerated by shock waves," Sov. Phys. JETP 44, 424 (1976).

${ }^{15}$ B. Vetter and B. Sturtevant, "Experiments on the Richtmyer-Meshkov instability on a air/ $\mathrm{SF}_{6}$ interface," Shock Waves 4, 247 (1995).

${ }^{16}$ D. J. Hill, C. Pantano, and D. I. Pullin, "Large-eddy simulation and multiscale modelling of a Richtmyer-Meshkov instability with reshock," J. Fluid Mech. 557, 29 (2006).

${ }^{17} \mathrm{O}$. Schilling and M. Latini, "High-order WENO simulations of threedimensional reshocked Richtmyer-Meshkov instability to late times: Dynamics, dependence on initial conditions, and comparisons to experimental data," Acta Math. Sci. 30, 595 (2010).

${ }^{18}$ E. Leinov, G. Malamud, Y. Elbaz, L. A. Levin, G. Ben-Dor, D. Shvarts, and O. Sadot, "Experimental and numerical investigation of the Richtmyer-Meshkov instability under re-shock conditions," J. Fluid Mech. 626, 449 (2009).

${ }^{19}$ V. V. Bashurov, Y. A. Bondarenko, V. I. Dudin, E. V. Gubkov, E. E. Meshkov, A. N. Poduvalov, A. A. Shanin, A. M. Stenin, V. A. Tilkunov, A. I. Tolshmyakov, L. Y. Trofimova, Y. V. Yanilkin, and V. V. Zmushko, "Experimental and numerical evolution studies for 2-D perturbations of the interface accelerated by shock waves," Proceedings of the Fifth International Workshop on the Physics of Compressible Turbulent Mixing, 1996.

${ }^{20}$ A. V. Smith, D. A. Holder, C. J. Barton, A. V. Morris, and D. L. Youngs, 
"Shock tube experiments on Richtmyer-Meshkov instability across a chevron profiled interface," Proceedings of the Eighth International Workshop on the Physics of Compressible Turbulent Mixing, 2001.

${ }^{21}$ D. A. Holder and C. J. Barton, "Shock tube Richtmyer-Meshkov experiments: Inverse chevron and half height," Proceedings of the Ninth International Workshop on the Physics of Compressible Turbulent Mixing, 2004.

${ }^{22}$ D. L. Youngs, in Implicit Large Eddy Simulation: Computing Turbulent Fluid Dynamics, edited by F. F. Grinstein, L. G. Margolin, and W. J. Rider (Cambridge University Press, Cambridge, 2007), pp. 392-412.

${ }^{23}$ D. Drikakis, F. Grinstein, and D. Youngs, "On the computation of instabilities and symmetry-breaking in fluid mechanics," Prog. Aerosp. Sci. 41, 609 (2005)

${ }^{24}$ D. A. Holder, A. V. Smith, C. J. Barton, and D. L. Youngs, "Shock-tube experiments on Richtmyer-Meshkov instability growth using an enlarged double-bump perturbation," Laser Particle Beams 23, 411 (2003).

${ }^{25}$ Implicit Large Eddy Simulation: Computing Turbulent Fluid Dynamics, edited by F. F. Grinstein, L. G. Margolin, and W. J. Rider (Cambridge University Press, Cambridge, 2007).

${ }^{26}$ D. Drikakis, "Advances in turbulent flow computations using highresolution methods," Prog. Aerosp. Sci. 39, 405 (2003).

${ }^{27}$ D. Drikakis and S. Tsangaris, "An implicit characteristic-flux-averaging method for the Euler equations for real gases," Int. J. Numer. Methods Fluids 12, 711 (1991).

${ }^{28}$ D. Drikakis and S. Tsangaris, "On the solution of the compressible NavierStokes equations using improved flux vector splitting methods," Appl. Math. Model. 17, 282 (1993).

${ }^{29}$ J. Zółltak and D. Drikakis, "Hybrid upwind methods for the simulation of unsteady shock-wave diffraction over a cylinder," Comput. Methods Appl. Mech. Eng. 162, 165 (1998).

${ }^{30}$ A. Bagabir and D. Drikakis, "Mach number effects on shock-bubble interaction," Shock Waves 11, 209 (2001).

${ }^{31}$ A. Bagabir and D. Drikakis, "Numerical experiments using highresolution schemes for unsteady, inviscid, compressible flows," Comput. Methods Appl. Mech. Eng. 193, 4675 (2004).

${ }^{32}$ D. Drikakis, M. Hahn, A. Mosedale, and B. Thornber, "Large eddy simulation using high-resolution and high-order methods," Philos. Trans. R. Soc. London, Ser. A 367, 2985 (2009).

${ }^{33}$ A. Mosedale and D. Drikakis, "Assessment of very high-order of accuracy in les models," ASME Trans. J. Fluids Eng. 129, 1497 (2007).

${ }^{34}$ B. Thornber, D. Youngs, D. Drikakis, and R. J. R. Williams, "The influence of initial conditions on turbulent mixing due to Richtmyer-Meshkov instability," J. Fluid Mech. 654, 99-139 (2010).

${ }^{35} \mathrm{~S}$. Tissera, V. Titarev, and D. Drikakis, "Chemically reacting flows around a double-cone, including ablation effects," AIAA Paper No. 2010-1285, 2010 .

${ }^{36} \mathrm{M}$. Hahn and D. Drikakis, "Large eddy simulation of compressible turbu- lence using high-resolution methods,” Int. J. Numer. Methods Fluids 47, 971 (2005)

${ }^{37}$ G. Allaire, S. Clerc, and S. Kokh, "A five-equation model for the simulation of interfaces between compressible fluids," J. Comput. Phys. 181, 577 (2002).

${ }^{38}$ A. W. Cook, "Enthalpy diffusion in multicomponent flows," Phys. Fluids 21, 055109 (2009).

${ }^{39} \mathrm{R}$. Abgrall, "How to prevent pressure oscillations in multi-component flow calculations: A quasi conservative approach," J. Comput. Phys. 125, 150 (1996).

${ }^{40} \mathrm{~S}$. K. Godunov, "A finite-difference method for the computation of discontinuous solutions of the equations of fluid dynamics," Mat. Sb. 47, 271 (1959).

${ }^{41}$ E. F. Toro, Riemann Solvers and Numerical Methods for Fluid Dynamics (Springer-Verlag, Cambridge, 1997).

${ }^{42}$ D. Drikakis and W. Rider, High-Resolution Methods for Incompressible and Low-Speed Flows (Springer Verlag, Cambridge, 2004).

${ }^{43} \mathrm{~B}$. Van Leer, "Towards the ultimate conservative difference scheme. IV. A new approach to numerical convection," J. Comput. Phys. 23, 276 (1977).

${ }^{44}$ K. H. Kim and C. Kim, "Accurate, efficient and monotonic numerical methods for multi-dimensional compressible flows. Part II: Multidimensional limiting process," J. Comput. Phys. 208, 570 (2005).

${ }^{45}$ B. Thornber, A. Mosedale, D. Drikakis, D. Youngs, and R. Williams, "An improved reconstruction method for compressible flows with low Mach number features," J. Comput. Phys. 227, 4873 (2008).

${ }^{46} \mathrm{~A}$. N. Kolmogorov, "The local structure of turbulence in an incompressible fluid at very high Reynolds numbers," Dokl. Akad. Nauk SSSR 30, 299 (1941).

${ }^{47}$ M. Hahn and D. Drikakis, "Implicit large-eddy simulation of swept wing flow using high-resolution methods," Int. J. Numer. Methods Fluids 47, 618 (2009)

${ }^{48}$ M. Hahn and D. Drikakis, "Assessment of large-eddy simulation of internal separated flow," ASME Trans. J. Fluids Eng. 131, 071201 (2009).

${ }^{49}$ B. Thornber, A. Mosedale, and D. Drikakis, "On the implicit large eddy simulation of homogeneous decaying turbulence," J. Comput. Phys. 226, 1902 (2007)

${ }^{50}$ B. Thornber, D. Drikakis, R. Williams, and D. Youngs, "On entropy generation and dissipation of kinetic energy in high-resolution shockcapturing schemes," J. Comput. Phys. 227, 4853 (2008).

${ }^{51}$ D. L. Youngs, "Three-dimensional numerical simulation of turbulent mixing by Rayleigh-Taylor instability," Phys. Fluids A 3, 1312 (1991).

${ }^{52}$ C. W. Barnes, S. H. Batha, A. M. Dunne, G. R. Magelssen, S. Rothman, R. D. Day, N. E. Elliott, D. A. Haynes, R. L. Holmes, J. M. Scott, D. L. Tubbs, D. L. Youngs, T. R. Boehly, and P. Jaanimagi, "Observation of mix in a compressible plasma in a convergent cylindrical geometry," Phys. Plasmas 9, 4431 (2002). 